\title{
Comparação entre as curvas de crescimento do Centers for Disease Control and Prevention e da Organização Mundial da Saúde para lactentes com idade de seis a 12 meses
}

\author{
Comparison between the Centers for Disease Control and Prevention and the World Health Organization \\ growth curves for six to 12 months old infants
}

\begin{abstract}
Bianca Sant'Anna Pires ${ }^{1}$, Rita de Cássia Akutsu², Lucíola de Castro Coelho ${ }^{3}$, Leiko Asakura4 , Anita Sachs ${ }^{5}$, Ana Cristina V. F. Abrão , Kelly Pereira Coca ${ }^{7}$
\end{abstract}

\section{RESUMO}

Objetivo: Comparar o estado nutricional de lactentes de ambos os sexos de acordo com a curva proposta pelo Centers for Disease Control and Prevention (CDC-2000) e com a nova curva de crescimento proposta pela Organização Mundial da Saúde (OMS-2006).

Métodos: Estudo longitudinal no qual foram acompanhadas crianças de ambos os sexos, nascidas a termo, com idade entre seis meses e um ano, em aleitamento materno predominante ou exclusivo. Os dados foram coletados por um único pesquisador por meio de ficha de atendimento nutricional composta por dados demográficos, antropométricos e sobre a alimentação da criança. Foram realizados testes de Wilcoxon e Friedman para comparar cada medida das curvas e analisar a variância, respectivamente.

Resultados: Da amostra final de 55 crianças, $51 \%(n=28)$ eram do sexo masculino. Os percentuais de eutrofia dos índices de peso por idade e de estatura adequada para idade de ambos os sexos apresentaram-se acima de $80 \%$. Não houve diferença significante na classificação do estado nutricional, segundo as curvas utilizadas. A porcentagem de concordância das duas curvas em relação ao peso e à estatura foi de 98,2 e $96,4 \%$, respectivamente.

Conclusões: As curvas de crescimento da OMS-2006 e do CDC-2000 foram similares para classificar o estado nutricional de lactentes entre 6-12 meses em aleitamento materno.

Palavras-chave: crescimento; antropometria; estado nutricional; criança.

\section{ABSTRACT}

Objective: Compare the nutritional status of male and female infants classified according to the growth curve proposed by the Center for Disease Control and Prevention (CDC-2000) and the new growth curve proposed by the World Health Organization (WHO-2006).

Methods: This longitudinal study enrolled children of 6-12 months old of both genders. They were born at term and exclusively or predominantly breastfed. A single researcher collected demographic, anthropometric and nutritional data. The Wilcoxon test was used to compare curves and the Friedman test was used to analyze the variance.
Instituição: Centro de Incentivo e Apoio ao Aleitamento Materno da Universidade Federal de São Paulo (Unifesp), São Paulo, SP, Brasil

${ }^{1}$ Mestranda em Nutrição pelo Departamento de Endocrinologia Clínica da Unifesp, São Paulo, SP, Brasil

2Doutora em Ciências da Saúde pela Universidade de Brasília (UnB); Professora do Departamento de Nutrição da UnB, Brasília, DF, Brasil

${ }^{3}$ Especialista em Nutrição Materno-Infantil pela Unifesp; Nutricionista do Departamento de Medicina Preventiva da Unifesp, São Paulo, SP, Brasil

${ }^{4}$ Doutora em Saúde Pública pela Universidade de São Paulo; Professora do Departamento de Medicina Preventiva da Unifesp, São Paulo, SP, Brasil ${ }^{5}$ Doutora em Ciências pela Unifesp; Professora do Departamento de Medicina Preventiva da Unifesp, São Paulo, SP, Brasil

${ }^{6}$ Doutora em Enfermagem pela Unifesp; Diretora do Centro de Incentivo e Apoio ao Aleitamento Materno/Banco de Leite Humano da Unifesp, São Paulo, SP, Brasil

${ }^{7}$ Mestre em Ciências pela Unifesp; Professora do Centro Universitário São Camilo, São Paulo, SP, Brasil
Endereço para correspondência:

Rita de Cássia Akutsu

Rua Borges Lagoa, 1.341, $1^{\circ}$ andar

CEP 04038-034 - São Paulo/SP

E-mail: rita_akutsu@yahoo.com.br

Conflitos de interesse: nada a declarar

Recebido em: 24/6/2009

Aprovado em: 5/3/2010 
Results: Among the 55 followed children, $51 \%(\mathrm{n}=28)$ were males. The percentages of normal weight-for-age and proper height-for-age in both genders were above $80 \%$. The nutritional status classification according to both growth curves was similar. Agreement between the two curves regarding weight and height was 98.2 and $96.4 \%$, respectively.

Conclusions: CDC-2000 and WHO-2006 growth curves were similar regarding nutritional status classification of 6-12 months old breastfeed infants.

Key-words: growth; anthropometry; nutritional status; child.

\section{Introdução}

As curvas de crescimento são um guia para monitorar o crescimento e desenvolvimento de crianças e norteiam condutas adequadas na orientação nutricional, essencial para implementação precoce da alimentação saudável.

A curva do National Center for Health Statistics (NCHS) foi adotada em 1977 pela Organização Mundial da Saúde (OMS) e passou a ser utilizada internacionalmente $e^{(1,2)}$. Posteriormente, o referencial antropométrico de crescimento do NCHS/1977 foi revisado e substituído. O novo referencial foi publicado pelo Centers for Disease Control and Prevention (CDC) em maio de 2000. As curvas do CDC, por sua vez, expressam um combinado de crianças alimentadas com fórmulas artificiais e leite materno. $\mathrm{O}$ CDC não recomendou um padrão de crescimento exclusivo para as crianças em aleitamento materno predominante porque não houve amostra suficiente nos Estados Unidos e a aplicação dessas curvas em crianças com aleitamento misto é complexa, em virtude das diferenças entre populações e indivíduos ${ }^{(1)}$.

Diante de divergências como a procedência do banco de dados, idade das crianças avaliadas, tipo de alimentação, entre outros fatores apontados por diversos estudos ${ }^{(3,4)}$, a OMS lançou em 2006 novas curvas para avaliar o crescimento de crianças de zero a cinco anos. O estudo para a elaboração das novas curvas foi populacional, com áreas de abrangências e critérios de elegibilidade bem definidos. A decisão de incluir populações das principais regiões do mundo apoiou-se na evidência de que os padrões de crescimento de pré-escolares bem nutridos e saudáveis são similares em diferentes países. Além disso, foram exigidos alguns critérios relacionados à alimentação das crianças, tais como: aleitamento materno exclusivo ou predominante por quatro meses, introdução de alimentos complementares aos seis meses e permanência em aleitamento materno até os 12 meses ${ }^{(2,5)}$.

Tendo em vista os objetivos do Cento de Incentivo e Apoio ao Aleitamento Materno da Universidade Federal de São Paulo (CIAAM/Unifesp), dentre eles, a prestação de serviço à comunidade na área do aleitamento materno e o acompanhamento do estado nutricional das crianças monitoradas, o presente estudo teve como objetivo comparar o estado nutricional de lactentes nascidos a termo de acordo com a curva proposta pelo $\mathrm{CDC}^{(6)}$ e a nova curva de crescimento proposta pela $\mathrm{OMS}^{(3)}$.

\section{Métodos}

Trata-se de um estudo longitudinal, no qual foram incluídos todos os lactentes de ambos os sexos, com idade entre seis meses e um ano, atendidos no CIAAM/Unifesp no segundo semestre do ano de 2007, que passaram por triagem com a equipe de enfermagem e realizaram, no mínimo, três consultas nutricionais. Todos os lactentes portadores de doenças que afetam o crescimento e o desenvolvimento foram excluídos do estudo e encaminhados para ambulatórios específicos. Incluíram-se apenas as crianças na referida faixa etária em virtude das características do atendimento do serviço de nutrição do CIAAM/Unifesp, que inicia o acompanhamento nutricional a partir do sexto mês.

Os dados foram coletados por um único pesquisador por meio de ficha de atendimento nutricional em cada consulta realizada, composta por dados demográficos, antropométricos e sobre a alimentação da criança (data de nascimento, sexo, peso e comprimento em cada consulta, duração e tipo de aleitamento materno).

Para aferir as medidas antropométricas, utilizou-se balança pediátrica digital (BP Baby, FilizolaTM), com capacidade/ divisão de $15 \mathrm{~kg} / 5 \mathrm{~g}$ para o peso, e antropômetro horizontal de madeira $\left(\operatorname{Carci}^{\circledR}\right)$ com escala de $0,1 \mathrm{~cm}$ e capacidade de $1 \mathrm{~m}$ para a estatura. As normas técnicas de mensuração foram aplicadas de acordo com o álbum de antropometria do Sistema de Vigilância Alimentar e Nutricional (Sisvan) ${ }^{(7)}$.

As variáveis analisadas no estudo foram sexo, idade, peso, estatura e permanência do aleitamento materno, além dos indicadores do estado nutricional peso para idade $(\mathrm{P} / \mathrm{I})$ e estatura para idade (E/I). Esses parâmetros são usualmente utilizados e podem ser associados entre si, fornecendo diferentes indicadores do estado nutricional da criança ${ }^{(8,9)}$.

Para análise dos dados antropométricos (peso e comprimento), utilizaram-se as curvas de crescimento 
(OMS e CDC) $)^{(3,6)}$, com apoio do software Anthro 2005. Os dados foram analisados por meio do programa Statistical Package for the Social Sciences (SPSS), versão 10.0. O estado nutricional foi definido de acordo com os pontos de corte estabelecidos pelo Sisvan ${ }^{(10)}$.

Para a análise dos resultados, foram aplicados os testes de Wilcoxon e Friedman ${ }^{(11)}$. O teste de Wilcoxon comparou cada medida das curvas de crescimento (OMS e CDC) $)^{(3,6)}$. A análise de variância de Friedman avaliou as três medidas efetuadas para a curva do $\mathrm{CDC}^{(6)}$ e para a curva da $\mathrm{OMS}^{(3)}$, separadamente. Fixou-se em $5 \%$ o nível de rejeição da hipótese de nulidade.

O estudo foi aprovado pelo Comitê de Ética em Pesquisa da Escola Paulista de Medicina da Unifesp, de acordo com as normas exigidas para pesquisas envolvendo seres humanos. A participação das crianças ocorreu mediante a assinatura do termo de consentimento livre e esclarecido pelo responsável.

\section{Resultados}

A amostra final correspondeu a 55 crianças. Houve uma perda de $14,1 \%$ em relação à população inicial $(n=64)$, referente às crianças que não completaram as três consultas de nutrição, exigidas no critério de inclusão deste estudo.

Todas as crianças residiam em domicílio de alvenaria, com água e esgoto encanados, e a maioria $(n=53)$, com mais de dois habitantes na casa. Dentre as mães, $36 \%$ possuíam o ensino médio completo, $33 \%$ o ensino fundamental incompleto, $16 \%$ o ensino fundamental completo, $7 \%$ o ensino médio incompleto, $6 \%$ o ensino superior incompleto e $2 \%$ não possuíam estudo. Do total de crianças avaliadas, 51\% $(\mathrm{n}=28)$ eram do sexo masculino. Permaneciam em aleitamento materno predominante ou exclusivo até o sexto mês $71,4 \%(n=20)$ dos lactentes masculinos e $81,5 \%(n=22)$ das meninas.

Em relação ao estado nutricional, no primeiro momento, avaliou-se por sexo. Quanto ao sexo masculino $(n=28)$, observou-se que, para o índice P/I, a maior parte das crianças encontrava-se eutrófica nas duas curvas e uma pequena porcentagem, com baixo peso (Gráfico 1) nos três momentos de aferição (CDC1 e OMS1 - primeira consulta, CDC2 e OMS2 - segunda consulta, CDC3 e OMS3 - terceira consulta). As aferições foram realizadas na primeira consulta e nas duas consultas subsequentes, apresentando um intervalo médio entre elas de 46 dias para os meninos e de 47 dias para as meninas, sendo descritas na Tabela 1. Para o índice E/I, observou-se que a maior parte dos meninos estava com a estatura adequada para a idade (Gráfico 2). Em ambos os casos, não houve diferença estatística significante entre as consultas

Das 27 crianças do sexo feminino, 81,5\% ( $\mathrm{n}=22)$ permaneciam em aleitamento materno até o sexto mês. $\mathrm{O}$ índice $\mathrm{P} / \mathrm{I}$ (Gráfico 1), indica uma pequena porcentagem de peso elevado nas duas curvas nas meninas, diferente do sexo masculino. Para o índice E/I, a maior parte das meninas estava com a estatura adequada para a idade (Gráfico 2). Analisando-se os dados do Gráfico 1, para o sexo feminino, não houve diferença estatística significante quanto ao estado nutricional entre as consultas.

Quanto aos testes estatísticos aplicados (Wilcoxon e Friedman), não houve diferença significante entre o diagnóstico do estado nutricional segundo as duas curvas. A porcentagem de concordância das duas curvas em relação ao peso foi de 98,2\%. Já para a estatura, a porcentagem de concordância foi de $96,4 \%$. Para ambos os sexos, observou-se uma prevalência $3 \%$ de baixo peso, $1,8 \%$ de peso elevado e $4,8 \%$ de baixa estatura.

\section{Discussão}

Reconhecer a importância da influência que as condições de vida exercem sobre o crescimento é fundamental. Por esse motivo, os principais órgãos internacionais preconizam o acompanhamento do crescimento como atividade de rotina na atenção à criança. Avaliações em diferentes etapas da vida

Tabela 1 - Medidas antropométricas dos lactentes atendidos no Centro de Incentivo e Apoio ao Aleitamento Materno da Universidade Federal de São Paulo

\begin{tabular}{lcrrrrr}
\hline Consulta & Medidas & Média & Mediana & Desvio padrão & P25 & P75 \\
\hline $1^{\mathrm{a}}$ & Peso $(\mathrm{g})$ & 7498 & 7250 & 1154 & 6730 & 8243 \\
& Estatura $(\mathrm{cm})$ & 66 & 66 & 4 & 64 & 68 \\
$2^{\mathrm{a}}$ & Peso $(\mathrm{g})$ & 7975 & 7815 & 1181 & 7150 & 8738 \\
& Estatura $(\mathrm{cm})$ & 68 & 68 & 4 & 65 & 71 \\
$3^{\mathrm{a}}$ & Peso $(\mathrm{g})$ & 8469 & 8170 & 1232 & 7568 & 9343 \\
& Estatura $(\mathrm{cm})$ & 70 & 71 & 4 & 67 & 73 \\
\hline
\end{tabular}

$\mathrm{P}$ : percentil. 


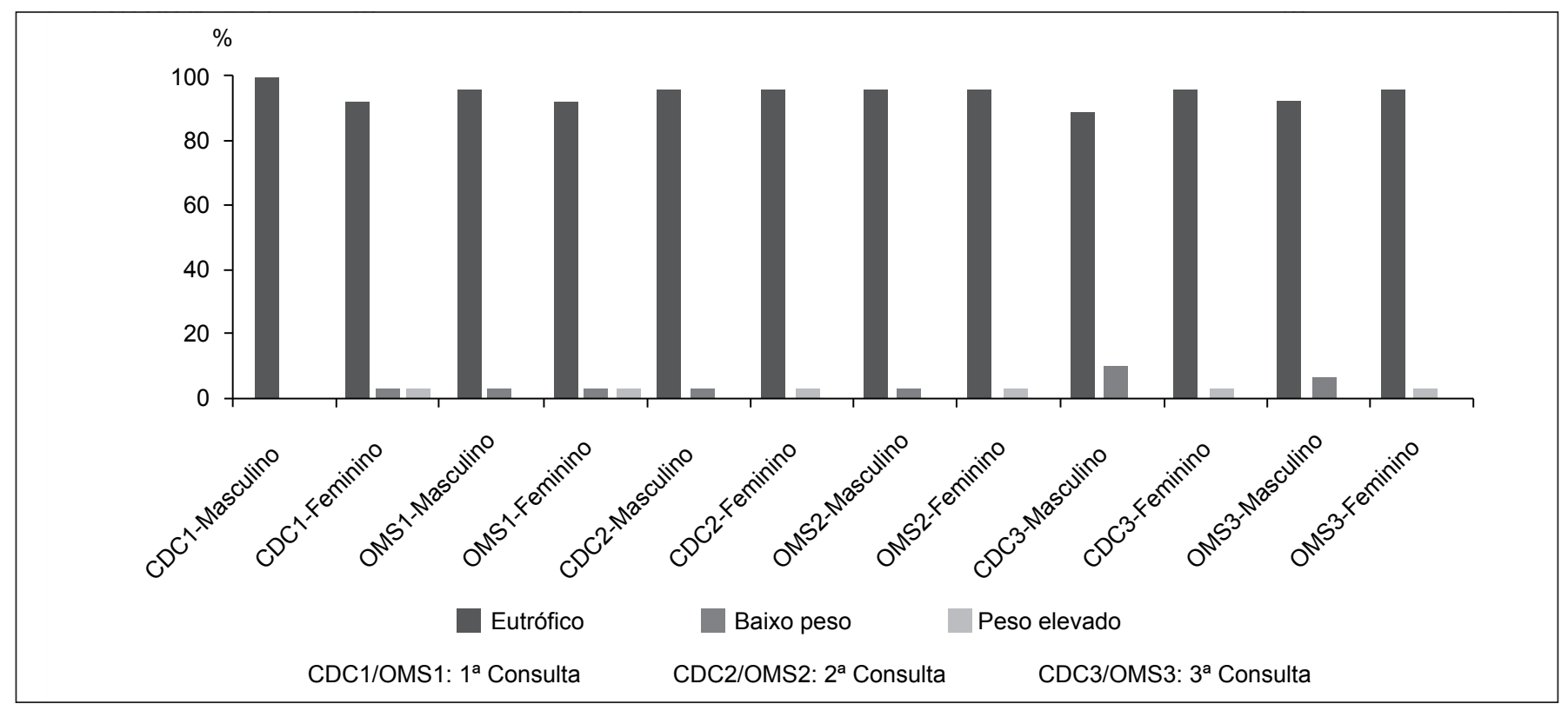

Gráfico 1 - Percentual de lactentes eutróficos, baixo peso e peso elevado nas curvas do Centers for Disease Control and Prevention (CDC) e da Organização Mundial da Saúde (OMS) para peso/idade.

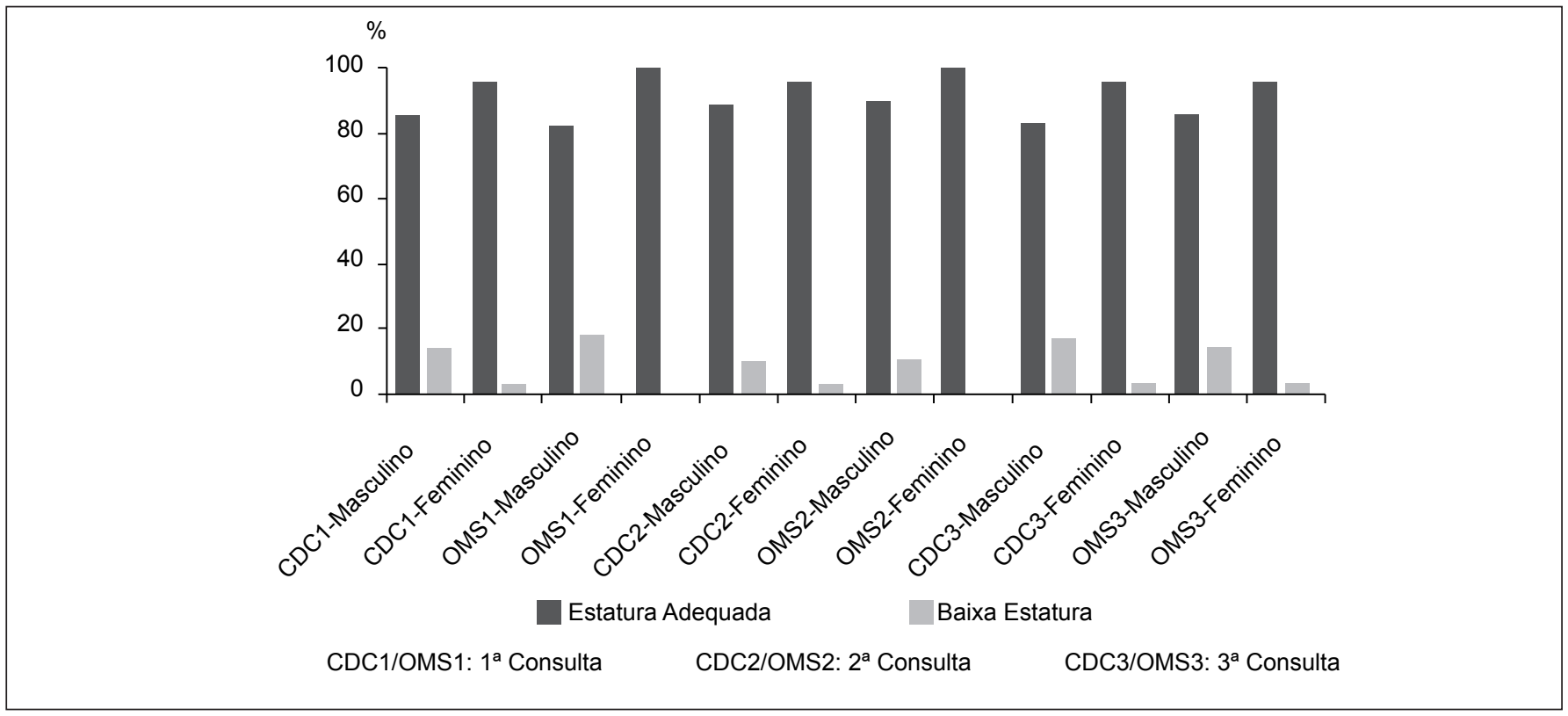

Gráfico 2 - Percentual de lactentes com estatura adequada e baixa estatura nas curvas do Centers for Disease Control and Prevention (CDC) e da Organização Mundial da Saúde (OMS) para estatura/idade.

podem contribuir para detectar riscos imediatos, mediatos ou tardios para saúde ${ }^{(12)}$.

Portanto, os estudos longitudinais são indicados para analisar efeitos de longo prazo na saúde. Ademais, os desfechos são registrados em momento próximo ao seu acontecimento, viabilizando a avaliação de agravos menos marcantes.
Observando-se os resultados obtidos, nota-se que, no presente estudo, não houve diferenças estatísticas significantes quando as duas curvas foram comparadas; além disso, a porcentagem de concordância entre elas é significante quanto aos resultados do diagnóstico do estado nutricional.

Esses resultados podem ter sido influenciados pelas características da amostra revelada na prevalência da permanência 
das crianças em aleitamento materno $(76,4 \%)$, quando comparada aos dados de estudos nacionais e do Ministério da Saúde ${ }^{(13,14)}$, em que a prevalência de aleitamento materno das crianças de São Paulo na faixa etária de 6-9 meses é de 45,5\% e, com 9-12 meses, 30,8\% ${ }^{(14)}$, o que parece indicar que as estratégias adotadas no CIAAM/Unifesp foram adequadas para a manutenção do aleitamento após os seis meses.

A sequência de eventos investigados permite que novas hipóteses sejam incorporadas, com o levantamento de dados específicos, como é o caso, por exemplo, da evolução do peso e estatura, o que viabiliza uma intervenção mais rápida ${ }^{(15)}$ e adequação das orientações nutricionais recomendadas aos cuidadores dessas crianças.

Lembrando que o primeiro ano de vida é mais delicado por influir no estado nutricional da criança durante a vida adul$\mathrm{ta}^{(10)}$, diagnosticar a obesidade precocemente, por exemplo, é essencial para propiciar uma vida adulta saudável. Deve-se levar em conta que os lactentes têm um alto ganho de massa gorda e são percebidos como saudáveis, mesmo quando estão acima do peso. Esses fatores devem ser lembrados e alertados para não confundir os pais das crianças ${ }^{(12)}$. Portanto, a escolha de instrumentos fidedignos contribui para uma orientação mais precisa por parte dos profissionais.

Onis e Victora ${ }^{(16)}$ destacam que as curvas são instrumentos valiosos para acompanhar o crescimento e o desenvolvimento das crianças, mas seria de suma importância a substituição da curva de crescimento do CDC por diversas questões, destacando-se os critérios estabelecidos quanto ao tipo de aleitamento. Onis $e t a l^{(17)} l e m b r a m$ que as novas curvas da OMS representam padrões normativos de crescimento baseados em crianças amamentadas, o que certamente levará à identificação mais precoce de sobrepeso e obesidade, problemas crescentes em todo o mundo.

Sabe-se que, para avaliar e monitorar o crescimento infantil, não basta aplicar um único método; faz-se necessário o acompanhamento do crescimento como um todo. Deve-se observar o crescimento infantil levando em conta seu grau de complexidade e todos os fatores que o envolvem. Portanto, o crescimento é um processo que deve ser monitorado constante e continuamente, por ser ininterrupto e veloz ${ }^{(18,19)}$ e, sempre que possível, devem-se produzir estudos com populações específicas, mormente as de maior risco, como os recém-nascidos pré-termo e os de baixa renda, como é caso de parte da população infantil brasileira.

Essas considerações vêm sendo tratadas por alguns autores. Entre eles, destaca-se o trabalho de Velho et al ${ }^{(20)}$ que, em um estudo transversal realizado em uma creche localizada na Zona Leste de São Paulo, analisaram o estado nutricional de 208 crianças de zero a cinco anos, buscando comparar as curvas de crescimento propostas pelo $\mathrm{CDC}^{(6)}$ e pela $\mathrm{OMS}^{(3)}$. A curva de crescimento proposta pela $\mathrm{OMS}^{(3)}$ demonstrou maior prevalência de risco de excesso de peso, segundo os indicadores $\mathrm{P} / \mathrm{E}$ e $\mathrm{P} / \mathrm{I}$, e maior prevalência de estatura maior e menor do que o esperado para a idade. Além disso, obteve-se maior prevalência de déficit de peso a partir dos indicadores da curva do $\mathrm{CDC}^{(6)}$, principalmente na população entre zero e dois anos.

Em outro estudo que comparou as duas curvas de crescimento de acordo com valores de Z-escore, Onis et a ${ }^{(17)}$ avaliaram uma amostra de 226 lactentes saudáveis amamentados. Os dados foram coletados em sete estudos na América do Norte e Norte da Europa. A partir dos resultados, concluiu-se que as diferenças são particularmente importantes durante a infância, principalmente para o indicador $\mathrm{P} / \mathrm{I}$. As divergências entre as curvas devem-se provavelmente ao tamanho e às características da amostra, à medida dos intervalos e ao tipo de alimentação.

Como já apontado no presente estudo, observou-se alta concordância entre as duas curvas, não havendo diferenças significantes. Esse fato pode ser justificado pelo pequeno número de crianças avaliadas, pela diferença de idade e tipo de aleitamento que esses lactentes recebiam. Com isso, a detecção das possíveis diferenças entre as curvas não foi possível. Tal fato reforça a necessidade de estudos com um número maior de crianças e faixas etárias mais bem definidas.

Outro fator favorável ao uso do novo parâmetro é que, em 2007, a OMS lançou as curvas de crescimento para avaliação das crianças acima de cinco anos e adolescentes. A atual referência preenche a lacuna antes existente nas curvas de crescimento, reforçando a utilização das novas curvas propostas pela $\mathrm{OMS}^{(7)}$.

O presente estudo teve como limitação o tamanho da amostra e o tempo de seguimento da população. Embora este trabalho tenha sido realizado com um percentual considerável da população atendida no CIAAM/Unifesp $(85,9 \%)$, não se pode fazer inferência para a população brasileira. Quanto ao tempo de seguimento, o ideal é que o acompanhamento se dê do nascimento ao final do primeiro ano de vida, o que certamente melhoraria a efetividade do trabalho realizado pela equipe multiprofissional do CIAAM/Unifesp.

Como já apontado, observou-se alta concordância entre as duas curvas, não havendo diferenças significantes e corroborando os achados do estudo realizado no Hospital Universitário de Brasília que, apesar de apresentar um tamanho amostral maior $(\mathrm{n}=119)$ e sugerir que a curva da OMS seja 
mais sensível para identificar baixa estatura, não mostrou diferenças significantes entre as curvas ${ }^{(2 \mathrm{r})}$.

Espera-se que os resultados aqui apresentados forneçam subsídios para uma melhor assistência aos usuários do CIAAM/
Unifesp, além de oferecer dados efetivamente produzidos com e para a população brasileira. É fundamental o apoio, a orientação e o acompanhamento das mães para que elas se sintam seguras durante a amamentação e a alimentação de seus filhos.

\section{Referências bibliográficas}

1. Soares NT. Um novo referencial antropométrico de crescimento: significados e implicações. Rev Nutr 2003;16:93-104.

2. Victora $\mathrm{CG}$, Araújo $\mathrm{CL}$, Onis $\mathrm{M}$ [homepage on the Internet]. Uma nova curva de crescimento para o século XXI [cited 2007 May 05]. Brasília: Ministério da Saúde; 2006. Available from: http://189.28.128.100/nutricao/docs/geral/ nova_curva_cresc_sec_xxi.pdf

3. WHO Multicentre Growth Reference Study Group. WHO Child Growth Standards based on length/height, weight and age. Acta Paediatr Suppl 2006;450:76-85.

4. WHO/UNICEF [homepage on the Internet]. Global strategy for infant and young child feeding [cited 2007 Aug 06]. Available from: http://www.who.int/nutrition/ publications/infantfeeding/9241562218/en/index.html

5. de Onis M, Garza C, Victora CG, Onyango AW, Frongillo EA, Martines J. The WHO Multicentre Growth Reference Study: planning, study design, and methodology. Food Nutr Bull 2004;25:S15-26.

6. CDC Growth charts: Unites States. Advance date from vital and health statistics 2000. Hyattsville, MD: National Center for Health Statistics; 2000.

7. Brasil. Ministério da Saúde [homepage on the Internet]. Novas curvas de avaliação de crescimento infantil. Classificação do estado nutricional [cited 2007 Aug 06]. Available from: http://nutricao.saude.gov.br/sisvan. php?conteudo=curvas_cresc_oms

8. Sigulem DM, Devincenzi MU, Lessa AC. Diagnóstico do estado nutricional da criança e do adolescente. J Pediatr (Rio J) 2000;76 (Suppl 3):S275-84.

9. Vitolo MR. Nutrição: da gestação ao envelhecimento. Rio de Janeiro: Rubio; 2008.

10. Brasil - SISVAN [homepage on the Internet]. Vigilância alimentar e nutricional - orientações básicas para a coleta, o processamento, a análise de dados e a informação em serviços de saúde [cited 2007 Aug 06]. Available from: http:// dtr2004.saude.gov.br/nutricao/documentos/orientacoes_basicas_sisvan.pdf
11. Siegel S, Castellan Jr NJ. Estatística não-paramétrica para ciências do comportamento. $2^{\text {nd }}$ ed. Porto Alegre: Artmed; 2006.

12. Zeferino AM, Barros Filho AA, Bettiol H, Barbieri MA. Monitoring growth. J Pediatr (Rio J) 2003;79 :S23-32.

13. Brasil. Ministério da Saúde. Prevalência de aleitamento materno nas capitais Brasileiras e no Distrito Federal. Brasília: Ministério da Saúde; 2001.

14. Lamounier JA. Tendências do aleitamento materno no Brasil. Rev Med Minas Gerais 1999;9:59-66.

15. Barros AJ, Santos IS, Victora CG, Albernaz EP, Domingues MR, Timm IK et al. The 2004 Pelotas birth cohort: methods and description. Rev Saude Publica 2006;40:402-13.

16. Onis M, Victora CG. Gráficos de crescimento para bebês alimentados com leite materno. J Pediatr (Rio J) 2004;80:85-7.

17. Onis M, Garza C, Onyango AW, Borghi E. Comparison of the WHO child growth standards and the CDC 2000 growth charts. J Nutr 2007;137:144-8.

18. Araújo CL, Victora CG, Hallal PC, Gigante DP. Breastfeeding and overweight in childhood: evidence from the Pelotas 1993 birth cohort study. Int J Obes (Lond) 2006;30:500-6.

19. Spyrides MH, Struchiner CJ, Barbosa MT, Kac G. The effect of breastfeeding practices on infant growth. Rev Bras Saude Mater Infant 2005;5:145-53.

20. Velho DI, Marçal IG, Teixeira AR, Simony RF. Evaluation of the nutritional state of children from 0 to 5 years old from a daycare center located in the city of São Paulo using the curves of growth. Nutrição em Pauta 2008;89:43-6.

21. Torres AA, Furumoto RA, Alves ED. Avaliação antropométrica de pré-escolares - comparação entre os referenciais: NCHS 2000 e OMS 2005. Rev Eletr Enf [serial on the Internet] 2007;9:166-75 [cited 2007 Aug 6]. Available from: http:// www.fen.ufg.br/revista/v9/n1/v9n1a13.htm 